\title{
A Semianalytical Model of the Synthetic Aperture, Interferometric Radar Altimeter Mean Echo, and Echo Cross-Product and Its Statistical Fluctuations
}

\author{
Duncan J. Wingham, Katharine A. Giles, Natalia Galin, Robert Cullen, \\ Thomas W. K. Armitage ${ }^{\circledR}$, and Walter H. F. Smith
}

\begin{abstract}
This paper develops a model of the synthetic aperture, interferometric satellite radar altimeter echo power, and echo cross-product. The model uses the smallness of the satellite pitch and roll angles, and the limited range of satellite altitude to provide a semianalytical echo model, whose numerical dimensions are limited to two in the synthetic aperture case or three in the interferometric case at large roll angles, making its application to extensive data practical. By not implementing the synthetic beam formation, it is demonstrated that the model recovers the conventional case of a pulse-limited altimeter. A theoretical description of the fluctuations in the multilooked, interferometric cross-product, and the synthetic aperture and pulse-limited powers is given by extending the model to describe correlations between individual looks in each case. The model offers the potential to retrieve ocean-surface parameters from synthetic aperture and pulse-limited altimeter data simultaneously, with obvious application to the new generation of altimeters onboard CryoSat-2, Sentinel-3, and Sentinel-6 during the transition to operational synthetic aperture radar altimetry.
\end{abstract}

Index Terms-Geophysical sea measurements, ocean surface, radar altimetry, radar remote sensing, spaceborne radar, synthetic aperture radar (SAR).

\section{INTRODUCTION}

$\mathbf{F}$ OR more than three decades, satellite altimetry has provided a wealth of information concerning the marine gravity field (see [1], [2]), the ocean dynamic topography (see [3]) and its variability (see [4], [5]), ocean tides (see [6]),

Manuscript received April 29, 2016; revised April 13, 2017 and September 7, 2017; accepted September 13, 2017. (Corresponding author: Thomas W. K. Armitage.)

D. J. Wingham was with the Centre for Polar Observation and Modelling, Department of Earth sciences, University College London, London WC1E 6BT, U.K. He is now with the Natural Environment Research Council, Swindon SN2 1EU, U.K.

K. A. Giles, deceased, was with the Centre for Polar Observation and Modelling, Department of Earth Sciences, University College London, London WC1E 6BT, U.K.

N. Galin was with the Centre for Polar Observation and Modelling, Department of Earth Sciences, University College London, London WC1E 6BT, U.K.

R. Cullen is with ESA-ESTEC, 2200 AG Noordwijk, The Netherlands (e-mail: Robert.Cullen@esa.int).

T. W. K. Armitage was with the Centre for Polar Observation and Modelling, Department of Earth Sciences, University College London, London WC1E 6BT, U.K. He is now with the Jet Propulsion Laboratory, California Institute of Technology, Pasadena, CA 91109 USA (e-mail: Tom.W.Armitage@jpl.nasa.gov).

W. H. F. Smith is with the NOAA Laboratory for Satellite Altimetry, College Park, MD 20740 USA (e-mail: walter.hf.smith@noaa.gov).

Color versions of one or more of the figures in this paper are available online at http://ieeexplore.ieee.org.

Digital Object Identifier 10.1109/TGRS.2017.2756854 the height of the ocean swell waves [7], and the ocean-surface wind speed [8]. Successive satellites, from Seasat (1978) onward, have had radar altimeters among their payload, and the use of radar altimeter observations in operational forecasting of ocean dynamics, waves, and winds is now familiar and well established [9]-[11]. While the intervening period has seen a continued improvement in the accuracy and precision of satellite altimeter measurements, arising variously from the improvement in gravity modeling and microwave tracking systems (see [12], [13]), increases in the pulserepetition frequency (PRF) [14], and the introduction of dual frequency measurements (see [14], [15]) and of solid-state power amplification [16], the design of radar altimeters has not altered greatly from that of Seasat [17], [18]: a normal incidence, full-deramp, linear frequency-modulated radar with a circular antenna of around $1-\mathrm{m}$ diameter operating at a central frequency of $13.6 \mathrm{GHz}$ with $320 \mathrm{-MHz}$ bandwidth. Similarly, the ocean-surface parameters derived from these measurements use, essentially, the model of the normal incidence scattering of the sea surface developed at the time and expressed in a closed form in [19].

Despite a design aimed at measuring the properties of the ocean surface, pulse-limited altimeters have also provided new insights into the behavior of the large ice sheets of Antarctica (see [20]) and Greenland [21], and, more recently, Arctic sea ice at the synoptic scale [22]. Nonetheless, the more variable topography of ice sheets, and the scattering inhomogeneity of sea ice interspersed with leads, has limited their performance and (along with the choice of orbit inclination) their coverage. For these reasons, the CryoSat-2 satellite [23], whose primary mission goal is to observe fluctuations in earth's marine and continental ice fields, has its main payload, the "SIRAL" altimeter, which differs in design from earlier, pulse-limited altimeters. It exploits a design used first in planetary satellite missions [24] that through improving the spatial resolution with along-track aperture synthesis, better distinguishes sea ice floes from the intervening leads, and as proposed in [25], adds a second altimeter in the across-track direction, with which the slopes of the marginal regions of the continental ice sheets can be determined.

However, it has been argued for some time [26], [27] that a synthetic aperture altimeter might also offer a greater measurement precision over the open ocean than does the pulse-limited design. By interrupting the synthetic aperture processing, it is possible to form the conventional pulse-limited echoes 
to examine the equivalence of the two operating modes, an important step in the transition toward the operational use of synthetic aperture radar (SAR) altimeters for oceanography. Based on CryoSat-2 SAR-mode acquisitions over the open ocean, this equivalence has been demonstrated (see [41]-[43]), and SAR altimeters are now becoming the standard for future open ocean applications as the ESA Sentinel-3 and Sentinel-6 satellites will both carry SAR altimeters. Further, the calibration of the CryoSat- 2 interferometer has demonstrated sufficient accuracy to measure the across-track slope of the ocean surface [28].

In this paper, we develop a robust model of the echo power and echo cross-product from a synthetic aperture, interferometric (SARIN mode) radar altimeter and demonstrate that by simple modifications, the pulse-limited model is obtained as a special case of the SAR-mode echo model. We take as a suitably accurate model of the SARIN echo cross-product, and SARIN and SAR mode echo powers, the description of [29], together with the modifications particular to CryoSat-2, such as the ellipticity of its antennas, given in [28]. As described there, however, that model would require numerical lookup tables of considerable size and dimensionality to apply in practice, and in Section II, the smallness of the satellite pitch and roll, and the limited range of satellite altitude are exploited to reduce the size of the lookup tables that are required in practice to two dimensions, or in the SARIN case, three dimensions. The model is developed around the CryoSat-2 altimeter parameters; however, the results are generally applicable to any SAR or SARIN altimeter instrument.

In Section III, we examine the precision, by which we mean the random fluctuation in the measurements arising from the presence of radar speckle. As with pulse-limited altimetry, this depends on the extent to which individual echoes that are gathered prior to multilooking are correlated. However, in addition, SAR (and SARIN) altimetry is distinct from pulse-limited altimetry in that the multilooking is also performed from individual echoes drawn from different statistical populations. In Section III, these aspects are examined through determining the effective number of looks - a measure of the effectiveness of the multilooking in suppressing speckle fluctuations-in the SARIN and SAR modes, and compared with that provided by pulse-limited operation. For this purpose, the models of Section II are extended to include the correlation properties of the echoes in addition to their means. Section III is concluded with a comparison of the modeled effective number of looks with that observed in practice.

Finally, three Appendixes provide a description of the data that were used in this paper (Appendix A), the details of the data processing we applied to obtain the results provided in the main text (Appendix B), and details of the effect of the altitude rate on the echo shape (Appendix C).

\section{Models of the Mean Echo Cross-Product AND ECHO POWER}

In general, the aim of SARIN altimeter measurements over the ocean is to determine the values of the ocean-surface height, parameterized by a delay time $\tau_{0}$ in the echo, the across-track gradient, parameterized by an "interferometer angle" $\theta$ in the interferometer echo cross-product (described more fully below), the ocean backscattering coefficient $\sigma^{0}$ (from which wind-speed is estimated) that depends on the received echo power, and the standard deviation of the surfaceheight distribution $\sigma_{s}$ (from which the significant wave height is estimated) obtained from the slope of the echo leading edge. To do so, samples of the echo power are fit to a model $p_{r}\left(\sigma^{0}, \sigma_{s} ; \tau-\tau_{0}\right)$ of the variation of echo power, or of the echo cross-product to a model $\Psi_{r}\left(\sigma^{0}, \sigma_{s}, \theta ; \tau-\tau_{0}\right)$ of the echo cross-product, by finding the values $\tilde{\tau}_{0}, \tilde{\sigma}^{0}, \tilde{\sigma}_{s}$, and $\tilde{\theta}$ of the parameters that minimize the weighted, least-squares difference between the observations and the model.

In addition to the ocean parameters, the echo power is also sensitive to parameters that characterize the measurement system (detailed in [28]) and which vary from echo to echo: the measurement altitude, the antenna pointing, generally in the case of CryoSat-2 requiring two angles to specify it, and, in the case of SAR and SARIN mode echoes, the velocity of the satellite. Thus, generally, the echo power is a 7-D or a 9-D function.

Were the models analytic, this presents no particular difficulty, but when, as is the case here, the model can only be "exactly" determined numerically, the high dimensionality presents a challenge in its computation if not its application. Thus, it is helpful if, to the greatest extent possible, the smallness of the variations in some parameters can be exploited to reduce the dimensionality of the functions that need numerical calculation. This is the aim of this section, in which we reduce the numerical dimensionality of the functions used in the SAR and pulse-limited echo models to two dimensions, and that for the SARIN echo or cross-product to three dimensions. We work by discussing first the SARIN echo cross-product model, and then, by successive simplifications, the SAR and finally pulse-limited models.

\section{A. Cross-Product Impulse Response}

In general, the mean echo cross-product $\Psi$ at near normal incidence from a rough surface is described by the triple convolution

$$
\Psi_{r}(\tau)=p_{t}(\tau) * p_{z}(\tau) * X(\tau, B)
$$

where $p_{t}$ is the compressed pulse shape (also known as the "single point-target response"), $p_{z}$ is the surface elevation probability density function, and $X$ is the cross-product "impulse response" of the surface [29]. $X$ is a function of delay and interferometer baseline $B$. If (1) is evaluated for zero baseline, the result is the echo power

$$
p_{r}(\tau)=p_{t}(\tau) * p_{z}(\tau) * X(\tau, 0) .
$$

The decomposition (2) is due to [19] who introduced the impulse response to describe the echo power; the extension of (1) to the echo cross-product involves no new assumptions.

The theoretical description of the interferometer echo crossproduct we use is that of [29], in the form tailored to the parameters of the SIRAL altimeter given in [28]. Fig. 1 shows the along- and across-track geometry of the radar imaging. 

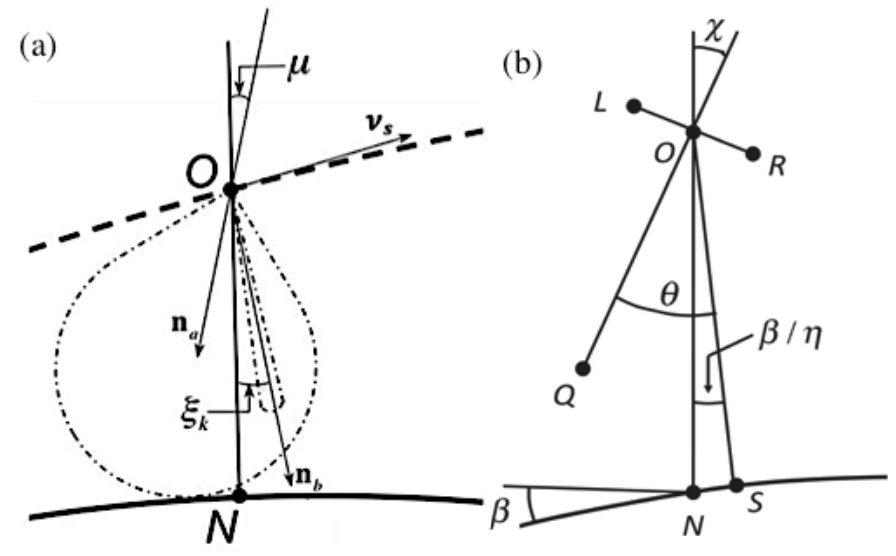

Fig. 1. (a) Along-track and (b) across-track geometry of the radar imaging. The satellite proceeds along its orbit [dashed line in (a)]. At any point on the orbit $O$, the satellite lies normally above the ellipsoidal nadir point $N$. The velocity vector $\boldsymbol{v}_{S}$ lies tangential to the orbit at $O$. The antennas' boresight direction $\boldsymbol{n}_{a}$ is generally pitched at an angle $\mu$ to the nadir direction. [When the satellite is pitched and rolled, as shown in (b), $\boldsymbol{n}_{a}$ has a component in the across-track direction.] Synthetic beams (only one is shown for clarity) are formed at a sequence of look angles $\xi_{k}$ that describe the angle between the synthetic beam boresight direction $\boldsymbol{n}_{b}$ and the nadir direction. In the across-track direction, the transmitting antenna ( $L$ for the SIRAL "A" chain used in this paper) falls on the left when viewed in the direction of flight. In SAR mode, the left antenna is used for reception; in SARIN mode, the left and right $(R)$ antennas are used for reception. The antennas' baseline normal direction $(O Q)$ is generally rolled by an angle $\chi$ with respect to the nadir direction. The "interferometer" angle $\theta$ can be interpreted geometrically as the angle of the first arrival, measured from the baseline normal, at the satellite of an echo from a surface with an across-track slope $\beta$ relative to the ellipsoid. Due to the earth's curvature, the surface angle perceived by the interferometer is smaller than $\beta$ by a factor $\eta$.

The multilooked cross-product impulse response from a surface slightly inclined to a sphere when using a mispointed, elliptical antenna is

$$
X(\tau, B) \sim \sum_{k=-\frac{(N-1)}{2}}^{\frac{(N-1)}{2}} X_{k}(\tau, B)
$$

where

$$
\begin{aligned}
& X_{k}(\tau, B) \\
& \sim \frac{\lambda^{2} G_{0}^{2} D_{0} c \sigma^{0}}{32 \pi^{2} h^{3} \eta} H\left(\tau+\frac{\eta h \xi_{k}^{2}}{c}\right) \\
& \cdot e^{-i k_{0} B\left(\chi+\frac{\beta}{\eta}\right)} \int_{0}^{2 \pi} d \vartheta d\left(\rho_{k} \cos \vartheta-\xi_{k}\right) e^{i k_{0} B \rho_{k} \sin \vartheta} \\
& \quad \cdot \exp \left[-2\left(\frac{\left(\rho_{k} \cos \vartheta-\mu-\varsigma / \eta\right)^{2}}{\gamma_{1}^{2}}+\frac{\left(\rho_{k} \sin \vartheta-\chi-\beta / \eta\right)^{2}}{\gamma_{2}^{2}}\right)\right] .
\end{aligned}
$$

$X_{k}$ are the cross-product impulse responses of individual beams. In (4), $\lambda$ is the carrier wavelength, $k_{0}$ is the carrier wavenumber, $c$ is the speed of light, and $h$ is the satellite altitude. $H$ is the Heaviside step function. $\eta=1+h / R$, where $R$ is the earth radius, is a geometric factor that arises because the surface is spherical rather than plane. Equation (4) employs the description

$$
G(\psi, \omega)=G_{0} \exp \left[-\psi^{2}\left(\frac{\cos ^{2} \omega}{\gamma_{1}^{2}}+\frac{\sin ^{2} \omega}{\gamma_{2}^{2}}\right)\right]
$$

for the antennas' gain pattern, in which $\psi$ is the polar angle measured from the antennas' boresight, $\omega$ is the azimuthal angle measured from the along-track direction, and $\gamma_{1}$ and $\gamma_{2}$ determine the along- and across-track width of the illumination. $\mu$ and $\chi$ are, respectively, the pitch and roll angles of the antennas (Fig. 1), and $\varsigma$ and $\beta$ are the along- and acrosstrack components of the surface vector gradient. $D_{0}$ is the one-way gain of a synthetic beam, and $d(\xi)$ is its pattern at an angle $\xi$ from its boresight in the along-track direction. $\xi_{k}$ is the along-track angle of the boresight of the $k$ th synthetic beam measured from the nadir direction (Fig. 1), termed the beam "look angle." Its value is

$$
\xi_{k}=k \pi /\left(N_{a} k_{0} v_{s} \Delta_{t}\right)
$$

where $N_{a}$ is the number of samples in the synthetic aperture, $k_{0}$ is the carrier wavenumber, $v_{s}$ is the magnitude of the satellite velocity in an earth-fixed frame, and $\Delta_{t}$ is the pulserepetition interval of the synthetic aperture. (Appendix A describes the transmission sequence in more detail.) In general, we denote the number of looks that form a multilooked echo by $N$. (To distinguish it later from a second measure, we will sometimes also describe $N$ as the "actual" number of looks.) In the SAR and SARIN modes, this is equal to the number of look angles, and in these cases, $N$ is given by

$$
N=\pi h \eta /\left(k_{0} v_{s}^{2} \Delta_{t} \Delta_{b}\right) .
$$

Here, $\Delta_{b}$ is the "burst" repetition interval, i.e., the interval between successive synthetic apertures. $\Delta_{b}$ takes different values in the SARIN and SAR modes so that the number of looks is not equal in the two modes, and we will distinguish these by $N_{S}$ and $N_{\mathrm{SI}}$ where necessary. In the pulse-limited case, the corresponding number of looks is $N_{P}=4 N_{a}$. $\rho_{k}$ in (4) is given by

$$
\rho_{k}=\sqrt{\frac{c \tau}{\eta h}+\xi_{k}^{2}}
$$

and accounts for the "slant range correction" (given by [29, eq. (27)]).

The dependence of the impulse response on the spacecraft attitude is of the form $X(\mu+\varsigma / \eta, \chi+\beta / \eta)$. This is a consequence of using small angle approximations for the attitude and surface gradient and retaining only the lead-order terms. For the same reason, (4) has no sensitivity to the spacecraft yaw angle. (See [29] for a detailed discussion.) The form arises because, to this level of approximation, the impulse response is not only separately sensible to the pitch and roll angles and the surface gradient, but also to their relative orientation: rolling the space craft has the same effect on the echo shape as tilting the surface. The sensitivity of the echoes to the along-track slope is negligible, and we shall generally suppose that $\varsigma=0$. This is not the case for the across-track slope, however, and we define the angle

$$
\theta=\chi+\beta / \eta \text {. }
$$

We term $\theta$ the "interferometer angle." For surfaces such as the ocean, this definition is consistent [28], with the geometric interpretation illustrated in Fig. 1(b), namely, that $\theta$ is the 
angle between the normal to the interferometer baseline and the direction of the point of closest range to the surface. With this definition, the dependence of the impulse response on the attitude becomes $X(\mu, \chi+(\beta / \eta)) \equiv X(\mu, \theta)$.

\section{B. Small Pitch Angle Form for the Cross-Product Impulse Response}

During nominal operation, the pitch and roll angles are small, of the order of $0.1^{\circ}$ or less. However, as part of this paper, we wish to apply the results to SARIN data acquired with larger roll angles, of the order of $1^{\circ}$. We, therefore, seek an expansion in small pitch angles of (4) to reduce the numerical complexity of the model of the cross-product, before proceeding to obtain expansions in small roll and pitch angles suitable for the SAR mode in Section II-C. The small pitch expansion is obtained by expanding the antenna gain in (4) and integrating term by term to obtain

$$
\begin{aligned}
\int_{0}^{2 \pi} & d \vartheta d\left(\rho_{k} \cos \vartheta-\xi_{k}\right) e^{i k_{0} B \rho_{k} \sin \vartheta} \\
& \cdot \exp \left[-2\left(\frac{\left(\rho_{k} \cos \vartheta-\mu\right)^{2}}{\gamma_{1}^{2}}+\frac{\left(\rho_{k} \sin \vartheta-\theta\right)^{2}}{\gamma_{2}^{2}}\right)\right] \\
\sim & I_{1}\left(\rho_{k} ; \theta, \xi_{k}\right)+\frac{4 \rho_{k} \mu}{\gamma_{1}^{2}} I_{2}\left(\rho_{k} ; \theta, \xi_{k}\right) \\
& +\mu^{2}\left(\frac{8 \rho_{k}^{2}}{\gamma_{1}^{4}} I_{3}\left(\rho_{k} ; \theta, \xi_{k}\right)-\frac{2}{\gamma_{1}^{2}} I_{1}\left(\rho_{k} ; \theta, \xi_{k}\right)\right)
\end{aligned}
$$

to second order in the pitch, where

$$
\begin{array}{r}
\left\{\begin{array}{l}
I_{1} \\
I_{2} \\
I_{3}
\end{array}\right\}=\int_{0}^{2 \pi} d \vartheta\left\{\begin{array}{c}
1 \\
\cos \vartheta \\
\cos ^{2} \vartheta
\end{array}\right\} d\left(\rho_{k} \cos \vartheta-\xi_{k}\right) e^{i k_{0} B \rho_{k} \sin \vartheta} \\
\cdot \exp \left[-\frac{2\left(\rho_{k} \sin \vartheta-\theta\right)^{2}}{\gamma_{2}^{2}}-\frac{2 \rho_{k}^{2} \cos ^{2} \vartheta}{\gamma_{1}^{2}}\right] .
\end{array}
$$

Substituting (10) into (4) and performing the summation in (3), one arrives at

$$
\begin{aligned}
& \mathrm{X}(\tau, B) \sim \frac{\lambda^{2} G_{0}^{2} D_{0} c \sigma^{0}}{32 \pi^{2} h^{3} \eta} \\
& \cdot e^{-i k_{0} B \theta}\left(\begin{array}{l}
H_{1}(\tau ; \theta)+\frac{\mu}{\gamma_{1}^{2}} H_{2}(\tau ; \theta) \\
+\mu^{2}\left(\frac{8}{\gamma_{1}^{4}} H_{3}(\tau ; \theta)-\frac{2}{\gamma_{1}^{2}} H_{1}(\tau ; \theta)\right)
\end{array}\right)
\end{aligned}
$$

where the definition of the functions in (12) is apparent from comparing their coefficients with the terms in (10).

\section{Small Pitch and Roll Angle Forms for the SARIN Mode, SAR Mode, and Pulse-Limited Power Impulse Response}

In the case of the SAR measurements used here, the interferometer angle $\theta$ is also small, so that one can expand the impulse response in terms of $\theta$ too. One has in place of (10)

$$
\begin{aligned}
\int_{0}^{2 \pi} & d \vartheta d\left(\rho_{k} \cos \vartheta-\xi_{k}\right) e^{-2\left(\frac{\left(\rho_{k} \cos \vartheta-\mu\right)^{2}}{\gamma_{1}^{2}}+\frac{\left(\rho_{k} \sin \vartheta-\theta\right)^{2}}{\gamma_{2}^{2}}\right)} \\
\sim & J_{1}\left(\rho_{k} ; \xi_{k}\right)+\frac{4 \rho_{k} \mu}{\gamma_{1}^{2}} J_{2}\left(\rho_{k} ; \xi_{k}\right) \\
& +\mu^{2}\left(\frac{8 \rho_{k}^{2}}{\gamma_{1}^{4}} J_{3}\left(\rho_{k} ; \xi_{k}\right)-\frac{2}{\gamma_{1}^{2}} J_{1}\left(\rho_{k} ; \xi_{k}\right)\right) \\
& +\theta^{2}\left(\frac{8 \rho_{k}^{2}}{\gamma_{2}^{4}} J_{4}\left(\rho_{k} ; \xi_{k}\right)-\frac{2}{\gamma_{2}^{2}} J_{1}\left(\rho_{k} ; \xi_{k}\right)\right)
\end{aligned}
$$

where now

$$
\begin{aligned}
&\left\{\begin{array}{c}
J_{1} \\
J_{2} \\
J_{3}, J_{4}
\end{array}\right\}=\int_{0}^{2 \pi} d \vartheta\left\{\begin{array}{c}
1 \\
\cos \vartheta \\
\cos ^{2} \vartheta, \sin ^{2} \vartheta
\end{array}\right\} d\left(\rho_{k} \cos \vartheta-\xi_{k}\right) \\
& \cdot \exp \left[-2 \rho_{k}^{2}\left(\frac{\cos ^{2} \vartheta}{\gamma_{1}^{2}}+\frac{\sin ^{2} \vartheta}{\gamma_{2}^{2}}\right)\right]
\end{aligned}
$$

and we have used the fact that in the SAR case, the baseline is zero. In contrast to the pitch $\mu$, (13) does not contain a linear term in the interferometer angle $\theta$. This is natural, in that, if the satellite is pitched nose-up, for example, the forward-looking synthetic beams have an increased weighting from the antenna pattern, the backward beams have a decreased weighting, and the reverse is true if the sign of the pitch changes. On the other hand, the echo shape in any particular beam is an even function with respect to the roll angle, i.e., is insensitive to the sign of the roll.

One may use the symmetry properties of the integrands in (14) to show that

$$
J_{2}\left(\rho_{k} ; \xi_{k}\right)=-J_{2}\left(\rho_{k} ;-\xi_{k}\right) .
$$

If the summation in (3) is formed symmetrically with respect to forward- and backward-looking beams, as indeed it is written, the consequence of (15) is that there is no linear dependence on the pitch in the multilooked echo. (If one wishes to measure the pitch, the behavior of individual beams is more sensitive than that of the multilooked echo. The distribution of power across the beams has been used to determine the satellite pitch [31].)

In detail, for the measurements we use, the summation is not exactly symmetric with respect to the forward and backward beams, and at high values of altitude rate, there is a correction that is needed to account for this. However, none of the altitude rates encountered in this paper are sufficient to warrant a correction. For completeness, the effect is described in Appendix C. In the remaining main text, we treat the summation as it appears in (3). Then, one has in place of (12)

$$
\begin{aligned}
X(\tau, 0) \sim & \frac{\lambda^{2} G_{0}^{2} D_{0} c \sigma^{0}}{32 \pi^{2} h^{3} \eta} \\
& \cdot\left(F_{1}(\tau)+\mu^{2}\left(\frac{8}{\gamma_{1}^{4}} F_{3}(\tau)-\frac{2}{\gamma_{1}^{2}} F_{1}(\tau)\right)\right. \\
\quad & \left.\theta^{2}\left(\frac{8}{\gamma_{2}^{4}} F_{4}(\tau)-\frac{2}{\gamma_{2}^{2}} F_{1}(\tau)\right)\right)
\end{aligned}
$$

for the SAR case, where the functions $F$ are defined in the obvious way. 
The pulse-limited case can be obtained in a similar fashion, by setting $D_{0}=d \equiv 1$ and $\xi_{k}=0$ in (4), which removes the synthetic beam formation and returns the problem to a pulselimited geometry. One then has a form sufficiently similar to (16) that we do not give it separately. In the pulse-limited case, the functions $F$ in (16) can be given analytical form by expanding the impulse response of [19] [see also (36)] and allowing for earth sphericity and the ellipticity of the antenna gain as has been done by Wingham and Wallis [32]. In this paper, we have not made use of these expressions because having necessarily to compute (16) numerically in the SAR case; it is then simple (and introduces least possibility of error) to set $D_{0}=d \equiv 1$ and $\xi_{k}=0$ and recompute the result.

\section{Power and Echo Cross-Product}

To determine the echo cross-product or power, the synthetic beams, the compressed pulse shape, and the surface roughness density function need a definite form. We describe these in this section. The SARIN-mode CryoSat-2 data that are available to us use a Hamming weighting when forming its synthetic beams, and in consequence, the synthetic beam pattern is

$$
\begin{array}{r}
D_{0} d(\phi)=\left(\sum_{n=0}^{N_{a}-1}\left(0.54-0.46 \cos \left(\frac{2 \pi n}{N_{a}-1}-\pi\right)\right)\right. \\
\left.\times e^{2 i k_{0} v_{s} \Delta_{t} \phi\left(n-\frac{N_{a}-1}{2}\right)}\right)^{2} .
\end{array}
$$

On the other hand, the SAR mode data uses a rectangular weighting, in which case

$$
D_{0} d(\phi)=\frac{\sin ^{2}\left(N_{a} \pi k_{0} v_{s} \Delta_{t} \phi\right)}{\sin ^{2}\left(\pi k_{0} v_{s} \Delta_{t} \phi\right)}
$$

In (17) and (18), $\phi$ denotes an angle measured from the maximum gain line of the beam. The compressed pulse of the SIRAL radar is accurately described by the function

$$
p_{t}(\tau)=p_{0} \operatorname{sinc}^{2}\left(\pi B_{c} \tau\right)
$$

where $B_{c}$ is the received bandwidth. While the specific form of the compressed pulse may differ from (19) for individual instruments, we adopt (19) as a general form of the altimeter compressed pulse shape and simply note that for specific instruments (19) may need to be modified, or an a posteriori correction applied to avoid potentially biasing ocean parameter retrievals. by

We use a Gaussian surface elevation density function given

$$
p_{z}(\tau)=\frac{1}{\sqrt{2 \pi} \sigma} e^{-\frac{1}{2}(\tau / \sqrt{2} \sigma)^{2}}
$$

where $\sigma=2 \sigma_{s} / c$ and the "significant wave height" ( $\mathrm{SWH}$ ) is equal to $4 \sigma_{s}$. Equation (20) is an approximation to the actual distribution of the ocean-surface elevations; it is possible, for example, to extend (20) by a term that accounts for the skewness of the height distribution in the manner of [33]. In fact, we have investigated doing so but we found that while the addition of a skewness term improved the least-squares error of the fit, it significantly degraded the precision of the range measurement, and in most cases, it was obvious even to the eye that the term was better fitting noise rather than better characterizing the ocean surface. Therefore, we have dropped this term in this paper.

With the convolutions of (1) performed, one obtains in the SARIN case, for example,

$$
\begin{aligned}
\Psi_{r}(\tau, B) \sim & \frac{\lambda^{2} G_{0}^{2} D_{0} c \sigma^{0}}{32 \pi^{2} h^{3} \eta} e^{-i k_{0} B \theta} \\
& \cdot\left(G_{1}\left(\tau ; \theta, \sigma_{s}\right)+\frac{\mu}{\gamma_{1}^{2}} G_{2}\left(\tau ; \theta, \sigma_{s}\right)\right. \\
& \left.\quad+\mu^{2}\left(\frac{8}{\gamma_{1}^{4}} G_{3}\left(\tau ; \theta, \sigma_{s}\right)-\frac{2}{\gamma_{1}^{2}} G_{1}\left(\tau ; \theta, \sigma_{s}\right)\right)\right)
\end{aligned}
$$

where the definition of the functions $G$ follows in the obvious way from (1) and (12). Similar forms are obtained for the SAR and pulse-limited cases.

\section{E. Effect of Variations in Satellite Altitude and Velocity}

Equation (21) and its analogous forms provide a description of the echo cross-product and power subject to small variations in the satellite pitch and, in the SAR and pulse-limited cases, roll. In this section, we describe how variations in the satellite altitude and velocity are accounted for.

The altitude of CryoSat-2 varies by some $50 \mathrm{~km}$ about $730 \mathrm{~km}$, and the power and cross-product have the first-order dependence on the altitude. While we have suppressed the dependence, the functions that appear in (21) are themselves functions of the altitude. The obvious approach to accounting for the variation is to return to (4), write $h=h_{0}+\delta$ and seek an expansion of the integral in powers of the variation of altitude $\delta$ about the fixed altitude $h_{0}$. This can be done in the pulse-limited case, but in the SARIN and SAR cases, the integrand is singular as $h_{0}+\delta \rightarrow-c \tau /\left(\eta \xi_{k}^{2}\right)$ as a result of (8), and its expansion does not converge there. However, this difficulty does not arise once the convolutions of (1) have been performed. Thus, we obtain the leading order term in $\delta$ by determining the numerical derivative of, for example, (21), with respect to $\delta$. In fact, it is only the derivative of $G_{1}$ that is needed; the higher order terms are small enough to be negligible. One then has

$$
\begin{aligned}
\Psi_{r}(\tau, B) & \\
\sim & \frac{\lambda^{2} G_{0}^{2} D_{0} c \sigma^{0}}{32 \pi^{2} h^{3} \eta} e^{-i k_{0} B \theta} \\
& \cdot\left(\begin{array}{l}
G_{1}\left(\tau ; \theta, \sigma_{s}\right)+\delta G_{4}\left(\tau ; \theta, \sigma_{s}\right)+\frac{\mu}{\gamma_{1}^{2}} G_{2}\left(\tau ; \theta, \sigma_{s}\right) \\
+\mu^{2}\left(\frac{8}{\gamma_{1}^{4}} G_{3}\left(\tau ; \theta, \sigma_{s}\right)-\frac{2}{\gamma_{1}^{2}} G_{1}\left(\tau ; \theta, \sigma_{s}\right)\right)
\end{array}\right)
\end{aligned}
$$

in place of (21), for example.

There are also small variations in the look angle sampling interval (6) and the number of looks (7) that occur through their dependence on the satellite altitude and velocity. In fact, (6) and (7) give time-averaged values; Equation (7), for 
example, does not generally provide an integer value for the number of looks $N$ in SARIN and SAR modes. In detail, the number of looks is an integer that varies by \pm 1 along the track in such a way as to provide the average value given by (7). However, the effect of the variations of these quantities on the echo impulse response is less than $0.25 \%$, which is undetectably small, and we, therefore, ignore it.

There is also a separate effect that arises when the direction of the satellite velocity is not tangential to the surface at the nadir point. The look angles of the synthetic beams are formed relative to the normal to the satellite velocity, and in the presence of an altitude rate, this results in an asymmetric distribution of beam look angles relative to the nadir direction, and in this case, the summation of (3) is no longer symmetric about $k=0$. However, the effect only becomes significant for high altitude rates, which were not observed in the data acquisitions used for this paper. For completeness, we give a more detailed description in Appendix C.

\section{ECHO FLuCtuAtions}

The echoes observed by the altimeter are subjected to radar speckle, and the purpose of the multilooking is to reduce the fluctuations due to speckle. Even after multilooking, the echoes suffer a residual fluctuation, and this fluctuation is the main source of noise on the measurements of the oceansurface parameters. A full description of the statistics of their residual fluctuation would require a fairly extensive Monte Carlo approach, and we have not attempted this. However, in all cases, the number of looks is fairly high, so the statistics of the multilooked echoes are close to Gaussian. In consequence, the statistics are reasonably described once the variance of the multilooked echoes is known. This section is concerned with the calculation of the variance and related quantities, and it is concluded with a comparison of the predicted echo fluctuation against those observed in practice.

\section{A. Effective Number of Looks}

Individual echoes $\psi$ have real and imaginary parts that are Gaussian distributed. Using $x^{*}$ to denote the conjugate of $x$, their power $p=\psi \psi^{*}$ is exponentially distributed [34]. The exponential distribution has the property that its variance equals the square of its mean. If $N$ independent echo powers are averaged to form a multilooked echo $q$, its variance is $\bar{p}^{2} / N$, where we use $\bar{x}$ to denote the mean of $x$, or, alternatively, $N=\bar{p}^{2} / \sigma_{q}^{2}$ where we use $\sigma_{x}^{2}$ to denote the variance of $x$. In the present case, however, the echoes we deal with are, in general, correlated and in addition, the mean echo varies from look to look. In consequence, the residual fluctuation is larger than one might suppose from simple considerations based on the number of looks $N$. We, therefore, follow [29] by introducing the effective number of looks $N_{e}$, which is the number of looks that would give rise to the actual residual fluctuation had the multilooked power been formed from independent echoes whose mean equals to that of the multilooked power.

In general, let us denote the covariance function between two random variables $a$ and $b$ as $C_{a b}$ and associate with it a correlation coefficient $R_{a b}=C_{a b} / \sigma_{a} \sigma_{b}$. Let $\psi$ denote an individual echo and let $p=\psi \psi^{*}$ denote the echo power. All the echoes we deal with are incoherent, i.e., their mean $\bar{\psi}=0$. In consequence, their variance $\sigma_{\psi}^{2}=\overline{\psi \psi^{*}}-\bar{\psi} \overline{\psi^{*}}=$ $\overline{\psi \psi^{*}}=\bar{p}$, i.e., their variance equals the mean power. Let $t=\psi_{1} \psi_{2}^{*}$ be the cross-product between two incoherent echoes $\psi_{1}$ and $\psi_{2}$ with equal mean powers $\bar{p}$. The covariance between the two echoes is then $C_{\psi_{1} \psi_{2}}=\overline{\psi_{1} \psi_{2}^{*}}-\overline{\psi_{1}} \overline{\psi_{2}^{*}}=\overline{\psi_{1} \psi_{2}^{*}}=\bar{t}$, and

$$
R_{\psi_{1} \psi_{2}}=\bar{t} / \bar{p} \text {. }
$$

In this context, the correlation coefficient is usually termed the coherence of the two echoes. $t$ has the probability density function [25]

$$
\begin{aligned}
& p_{\psi_{1} \psi_{2}}(z) d z=\frac{2}{\pi \bar{p}^{2}\left(1-R_{\psi_{1} \psi_{2}}^{2}\right)} K_{0}\left(\frac{2|z|}{\bar{p}\left(1-R_{\psi_{1} \psi_{2}}^{2}\right)}\right) \\
& \times \exp \left(\frac{2 R_{\psi_{1} \psi_{2}} \mathcal{R}(z)}{\bar{p}\left(1-R_{\psi_{1} \psi_{2}}^{2}\right)}\right) .
\end{aligned}
$$

Here, $\mathcal{R}(z)$ describes the real part of $z$. From this density function, one may obtain the result $\overline{t t^{*}}=\bar{p}^{2}\left(1+R_{\psi_{1} \psi_{2}}^{2}\right)$. One thus has $\sigma_{t}^{2}=\overline{t t^{*}}-\overline{t t^{*}}=\bar{p}^{2}$, i.e., the variance of the crossproduct is simply the square of the mean power. However, since $\overline{t t^{*}}=\overline{\psi_{1} \psi_{2}^{*} \psi_{2} \psi_{1}^{*}}=\overline{\psi_{1} \psi_{1}^{*} \psi_{2} \psi_{2}^{*}}=\overline{p_{1} p_{2}}$, one also has

$$
C_{p_{1} p_{2}}=\overline{p_{1} p_{2}}-\bar{p}^{2}=\bar{p}^{2} R_{\psi_{1} \psi_{2}}^{2}=C_{\psi_{1} \psi_{2}}^{2} .
$$

This is an extremely useful result, in that it permits us to determine the covariance of the power in terms of the covariance of the field. (This result is not original; it appears, for example, in a rather more general setting in [35].) In the event that $\psi_{1}=\psi_{2}, t=p, R_{\psi_{1} \psi_{2}}=1$, (24) reverts to the familiar exponential distribution for the power of an incoherent echo, and (25) simply expresses the familiar result that the variance of the power equals the mean power squared. (It is interesting to note that as we do following (24), that this result holds in the more general case of a partially coherent cross-product.)

Let the multilooked cross-product or power be the sum of $N$ individual looks $q=(1 / N) \sum_{n=0}^{N-1} t_{n}$, where $t_{n}$ denotes an individual look. We define the effective number of looks to be $N_{e}=\bar{q} \overline{q^{*}} / \sigma_{q}^{2}$. Then

$$
\begin{aligned}
\sigma_{q}^{2}=\overline{(q-\bar{q})^{2}} & =\frac{1}{N^{2}} \sum_{n=0}^{N-1} \sum_{m=0}^{N-1} \overline{\left(t_{n}-\overline{t_{n}}\right)\left(t_{m}-\overline{t_{m}}\right)} \\
& =\frac{1}{N^{2}} \sum_{n=0}^{N-1} \sum_{m=0}^{N-1} \overline{p_{n}} \overline{p_{m}} R_{t_{n} t_{m}}
\end{aligned}
$$

and thus, in general

$$
N_{e}=\frac{\left|\sum_{n=0}^{N-1} \overline{t_{n}}\right|^{2}}{\sum_{n=0}^{N-1} \sum_{m=0}^{N-1} \overline{p_{n} p_{m}} R_{t_{n} t_{m}} .}
$$

In the event that we are concerned with multilooked powers, rather than the cross-product, one has $\overline{t_{n}} \overline{t_{m}^{*}}=\overline{p_{n}} \overline{p_{m}}$ in the denominator on the right-hand side of (27). From (27), one has in general that $N_{e} \leq N$. 
In Sections III-B-III-E, the statistics of the echoes in the SAR, SARIN, and pulse-limited cases is considered separately to arrive at simpler representations in each of these cases.

\section{B. Independence of the Looks in SAR Mode}

In the SAR case, we are dealing only with powers, and the mean powers $\overline{p_{n}}$ that then appear in the denominator and numerator of (27) are those obtained by applying (1) to the individual impulse responses of (4). The correlation coefficient in (27) is then the correlation that occurs between different look angles. In the level-1b (L1b) processor, each look comes from a different burst, and the principle mechanism that gives rise to decorrelation is the spatial fading that arises from the displacement of the antenna between looks. At least for neighboring looks, the change in angle between looks is very small and has little effect on the echo power, and the look angle is adjusted so that each look illuminates the same area of surface. The decorrelation can then be examined by considering the covariance between the echo at a given look angle and that which would be observed when the antenna is displaced along-track by a small horizontal distance $x$, i.e., we consider the covariance function $C_{k}(\tau, x)$, where $k$ denotes the look angle through (6).

In this case, the covariance can be obtained by following a similar path as [29] (see also [36]). $C_{k}(\tau, x)$ is given by a similar triple convolution to (1), namely,

$$
C_{k}(\tau, x)=p_{t}(\tau) * p_{z}(\tau) * \Pi_{k}(\tau, x)
$$

where, in this case, the covariance impulse response is given by

$$
\begin{aligned}
\Pi_{k}\left(\tau, \xi_{k}, x\right) \sim & \frac{\lambda^{2} G_{0}^{2} D_{0} c \sigma^{0}}{32 \pi^{2} h^{3} \eta} H\left(\tau+\frac{\eta h \xi_{k}^{2}}{c}\right) \\
& \cdot \int_{0}^{2 \pi} d \vartheta d\left(\rho_{k} \cos \vartheta-\xi_{k}\right) e^{2 i k_{0} x \cos \vartheta} \\
& \cdot \exp \left[-2 \rho_{k}^{2}\left(\frac{\cos ^{2} \vartheta}{\gamma_{1}^{2}}+\frac{\sin ^{2} \vartheta}{\gamma_{2}^{2}}\right)\right] .
\end{aligned}
$$

It may be noted that the expression for $\Pi_{k}$ is essentially the same as that of $X_{k}$, save that the phase difference in (4) that arises between the two antennas displaced across-track is replaced in (29) with the phase difference that occurs between the same antenna at two different locations along the track, and a factor of 2 appears because, unlike the interferometer, path differences occur on transmission and reception. In (29), we have also assumed nadir antenna pointing, ignoring the small effect of pitch and roll on the covariance function.

With the covariance obtained from (28) and (29), and the power of an individual look obtained from (1) using (4) with $B=0$, the correlation function may be computed. In Fig. 2, we show the power correlation coefficient for various look angles at $\tau=\eta h \xi_{k}^{2} / c$ as a function of the along-track separation $x$. (The numerical values we have used in this and future calculations are given in Table II.) The top panel of Fig. 2 shows the correlation functions at zero SWH; the bottom panel of Fig. 2 shows the correlation functions with
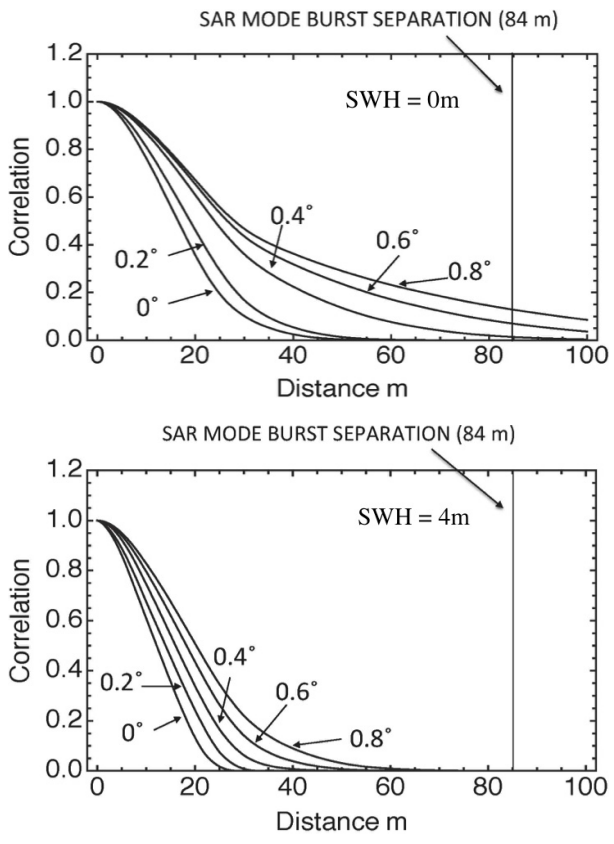

Fig. 2. Power correlation coefficient for a sequence of look angles as a function of the along-track separation of the looks at (Top) zero SWH and (Bottom) 4-m SWH. Also shown is the along-track separation of the bursts in SAR mode of $84 \mathrm{~m}$. In SARIN mode, the burst separation is $336 \mathrm{~m}$.

an SWH of $4 \mathrm{~m}$. At low wave heights and small look angles, the power decorrelates over some $20 \mathrm{~m}$. This is the expected result for a beam-limited system, in that the decorrelation distance is approximately equal to the synthetic aperture length, which in this case is $26 \mathrm{~m}$. At higher look angles, however, the beams become pulse-limited along-track, and the decorrelation length correspondingly increases.

Marked on Fig. 2 is the spatial separation of the bursts in SAR mode, $84 \mathrm{~m}$. It can be seen that at this separation, most of the beams at zero wave height will have decorrelated, although some correlation remains at high look angles. With appreciable $\mathrm{SWH}$, however, all of the beams decorrelate, exhibiting a beam-limited behavior. This occurs because the effect of the wave height is to extend the pulse-limited area to the extent that the synthetic beams become beam, and not pulse, limited in the along-track direction. However, even when SWH is low, the beams that remain correlated have very little weight in the multilooking, because they are heavily weighted by the antenna pattern. Thus, in practice, it is a good approximation to assume that the looks are independent in SAR mode. One, therefore, has $R_{t_{n} t_{m}}=\delta_{m, n}$ and (27) simplifies to

$$
N_{e}^{(S)}=\frac{\left|\sum_{m=0}^{N_{S}-1} \overline{p_{m}}\right|^{2}}{\sum_{m=0}^{N_{S}-1}{\overline{p_{m}}}^{2}}
$$

in SAR mode.

\section{Correlation Between the Powers at the Interferometer Antennas and the Coherence of the Echo Cross-Product}

In SARIN mode, the burst separation is larger than that in the SAR mode, some $336 \mathrm{~m}$, and the conclusion that the echoes in each beam are uncorrelated one from another carries 
over from the SAR mode. In the case of the echo crossproduct, one then has

$$
N_{e}^{(\mathrm{SI} c)}=\frac{\left|\sum_{m=0}^{N_{\mathrm{SI}}-1} \overline{p_{m}} R_{\mathrm{LR}}(m)\right|^{2}}{\sum_{m=0}^{N_{\mathrm{SI}}-1}{\overline{p_{m}}}^{2}}
$$

where we have made use of (23), and we denote by $R_{\mathrm{LR}}(m)$ the correlation, or coherence, between the cross-product of the echoes at the left and right antennas (see Fig. 1).

If, on the other hand, we consider the echo power in the SARIN mode, determined by summing the power over the beams, as is the case for SAR mode, at each antenna, and then summing the result for each antenna

$$
N_{e}^{(\mathrm{SI} p)}=\frac{2\left|\sum_{m=0}^{N_{\mathrm{SI}}-1} \overline{p_{m}}\right|^{2}}{\sum_{m=0}^{N_{\mathrm{SI}}-1} \bar{p}_{m}^{2}\left(1+R_{\mathrm{LR}}^{2}(m)\right)}
$$

where we have made use of (25) to express the correlation between the echo powers at the two antennas in terms of the coherence of their fields. The number of looks, $2 N_{\mathrm{SI}}$, is doubled when using the SARIN echo power because the measurements at both antennas are incoherently summed. Thus, the same correlation function appears in both the expressions for the multilooked cross-product and the multilooked power, but with opposite effect: a high correlation results in a high number of effectively independent looks in the case of the cross-product, but has the effect of reducing the number of effectively independent looks in the case of the multilooked power.

In this case, the covariance function impulse response is that of the echoes arriving at the right and left antennas. The corresponding covariance impulse response is simply that of (4), and the power impulse response also given by (4) with $B=0$. Fig. 3 shows the power correlation function of the echoes at the two antennas at two look angles as a function of echo delay time $\tau+\eta h \xi_{k}^{2} / c$. The top panel of Fig. 3 shows the coherence for zero SWH; the bottom panel of Fig. 3 shows the coherence at 4-m SWH. It can be seen that at zero echo delay, the correlation remains high in all cases. Even at high wave heights, the correlation exceeds 0.7 , while for small wave heights and beam look angles, the correlation is close to 1 . Since the height precision is most sensitive to the echo in the vicinity of zero delay time, it is apparent that summing the echo powers from the two antennas will not provide much improvement in the height precision over that obtained from an echo from a single antenna.

Except for negative delays, the correlation is only weakly dependent on the look angle, and at negative delays, the differences are those occurring at very low signal powers, where, in practice, noise and other small instrumental disturbances occur. (The oscillatory character of the $0^{\circ}$ look angle plot in the zero wave-height case in the top panel of Fig. 3 is the result of the single point-target impulse response in (19). For other values of look angle or wave height, these are smoothed out either by the spreading in delay time of the echo, or the wave-height distribution.) Thus, one can make the reasonable approximation to the effective number of looks of (32) that
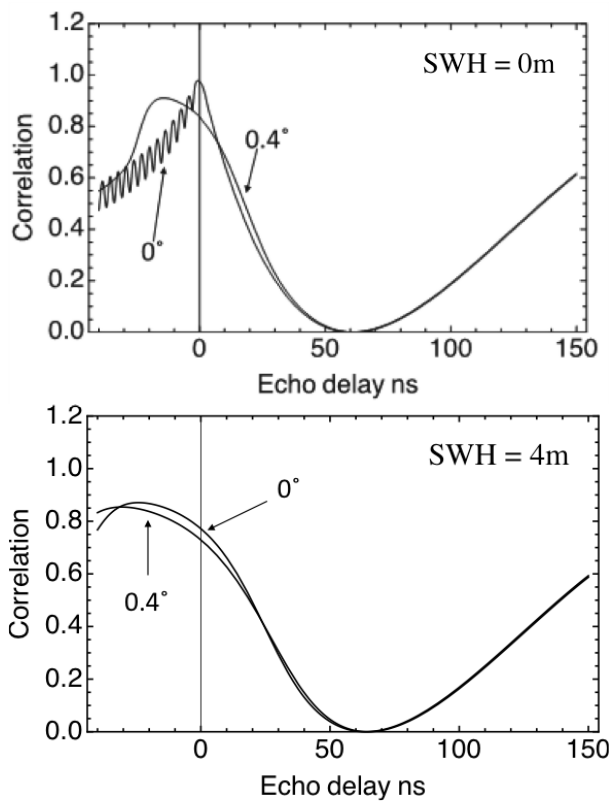

Fig. 3. Power correlation coefficient between the echoes at the two antennas of the interferometer for two look angles at (Top) zero SWH and (Bottom) 4-m SWH. Note that the power correlation coefficient is the square of the modulus of the coherence. The oscillatory character of the $0^{\circ}$ incidence beam at zero SWH is the result of the single point-target response; at higher SWH or look angles, this oscillation is washed out by the convolution of (1).

for the SARIN mode multilooked power

$$
N_{e}^{(\mathrm{SI} p)}=\frac{2\left|\sum_{m=0}^{N_{\mathrm{SI}}-1} \overline{p_{m}}\right|^{2}}{\left(1+R_{\mathrm{LR}}^{2}(0)\right) \sum_{m=0}^{N_{\mathrm{SI}}-1} \bar{p}_{m}^{2}} .
$$

The correlation functions in Fig. 3 also have a separate relevance in that they are the square of the coherence of the beam cross-product, and in this case, a high coherence results in a large effective number of looks. From Fig. 3, one can see that the effective number of looks will be high in the vicinity of zero delay time, but will fall as the delay time increases. We shall find this reduction in the effective number of looks apparent in the measured cross-product phase noise.

\section{Correlation Between the Powers of the \\ Pulse-Limited Looks}

In contrast to the SARIN mode and SAR mode cases, the multilooking of the pulse-limited echoes sums over echoes drawn from the same mean, so that $\overline{p_{n}}=\overline{p_{m}}$ in (27), which in consequence simplifies to

$$
N_{e}^{(\mathrm{PL})}=\frac{N_{P}^{2}}{\sum_{n=0}^{N_{P}-1} \sum_{m=0}^{N_{P}-1} R_{p_{n} p_{m}}} .
$$

In this case, $N_{e}^{(\mathrm{PL})}<N_{P}$ if the individual looks are correlated with one another.

In the pulse-limited case, the corresponding covariance function is again that resulting between two echoes observed with a small along-track displacement $x$. As is the case for the pulse-limited power, one may obtain this covariance function by setting $D_{0}=d \equiv 1$ and $\xi_{k}=0$ in (29) to 


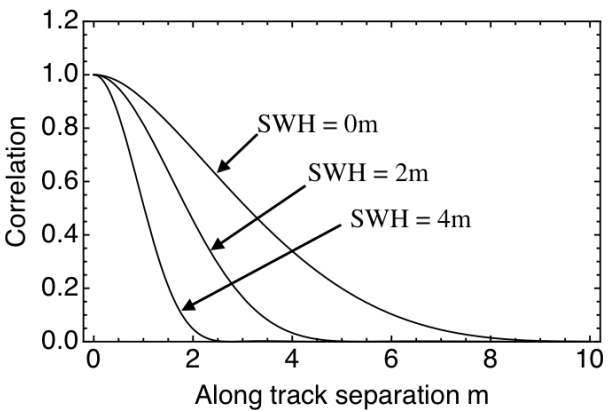

Fig. 4. Power correlation coefficient of the pulse-limited echoes at zero delay time for wave heights of 0,2 , and $4 \mathrm{~m}$, as functions of the alongtrack separation of the echoes. Increasing wave height corresponds to more rapid decorrelation. When pulse-limited echoes are formed from SAR mode operation, the separation of the echoes is $0.41 \mathrm{~m}$.

obtain

$$
\begin{aligned}
\Pi(\tau, x) \sim \frac{\lambda^{2} G_{0}^{2} c \sigma^{0}}{32 \pi^{2} h^{3} \eta} H(\tau) \cdot \int_{0}^{2 \pi} d \vartheta e^{2 i k_{0} x \cos \vartheta} \\
\quad \times \exp \left[-2\left(\frac{c \tau}{h \eta}\right)\left(\frac{\cos ^{2} \vartheta}{\gamma_{1}^{2}}+\frac{\sin ^{2} \vartheta}{\gamma_{2}^{2}}\right)\right]
\end{aligned}
$$

for the covariance impulse response. For small ellipticity, following the method of [32], this integral can be given the approximate form:

$$
\Pi(\tau, x) \sim \frac{\lambda^{2} G_{0}^{2} \sigma^{0} c}{16 \pi h^{3} \eta} \cdot e^{-\left(\frac{2 c \tau}{h \eta \bar{\gamma}^{2}}\right)} \cdot J_{0}\left(2 k_{0} x \sqrt{\frac{c \tau}{h \eta}}\right)
$$

where $J_{0}(z)$ denotes a Bessel function of the first kind, and $2 / \bar{\gamma}^{2}=1 / \gamma_{1}^{2}+1 / \gamma_{2}^{2}$. If one ignores the ellipticity, (36) is the result of [36]. If one sets $x=0$ in (36), one obtains the pulselimited power impulse response. Equation (36) represents the expression for the impulse response of [19], allowing for the curvature of the earth and the small ellipticity of the antennas [32]. (While we have used a numerical integration of (35) to obtain the results in this section, we have used (36) to verify the numerical integrations.)

Fig. 4 shows the power correlation coefficient for the pulselimited echoes at zero delay time and for three different wave heights. In a burst, the echoes are separated along-track by $0.41 \mathrm{~m}$. It can be seen that at all wave heights, successive echoes in the burst are highly correlated, and even at high wave heights, only every fifth echo is completely decorrelated at zero echo delay. The rates of decorrelation in Fig. 4 are in qualitative agreement with those suggested previously by a more approximate argument [37], [38].

\section{E. Comparison of the Predicted and Measured Effective Number of Looks}

We close this section with a comparison of the calculated effective number of looks $N_{e}$ for the SARIN mode crossproduct, the SAR mode power, and the pulse-limited power. (The results of the SARIN-mode power are essentially the same as those of the SAR mode.) A description of what determines the number of looks in each mode and how the multilooking is performed in practice in the three cases is given in Appendix A. In the following, we make comparisons

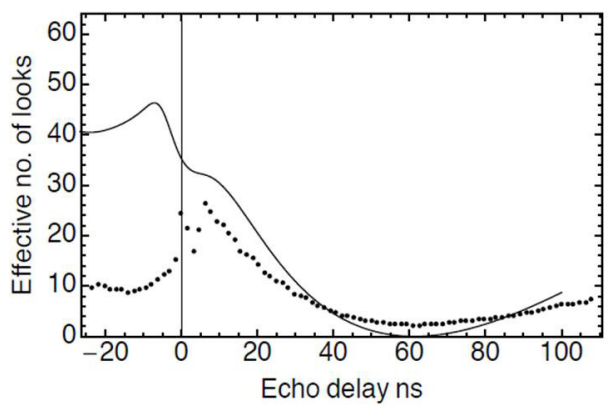

Fig. 5. Comparison of the calculated (solid line) and observed (dots) effective number of looks for SARIN-mode echo cross-products at $20 \mathrm{~Hz}$. The calculated curve is for a wave height of $2 \mathrm{~m}$. The actual number of looks at $20 \mathrm{~Hz}$ is 61 .

of echoes at the $20-\mathrm{Hz}$ rate, which is the highest rate at which echoes are available in all three data sets.

Fig. 5 shows a comparison between the effective number of looks $N_{e}^{(\mathrm{SIc})}$ of the SARIN echo cross-product calculated from (31) with the observed value at the $20-\mathrm{Hz}$ rate. It can be seen immediately that the effective number of looks $N_{e}^{(\text {SIc) }}$ is considerably smaller than the actual number of looks $N_{\mathrm{SI}}$ : for positive delay times, both the calculated and the observed effective number of looks are less than half the actual number of looks, which is 63 in this case. This is the result of forming multilooked echoes drawn from populations with different means. Because some echoes have a higher power than others, so too do their speckle fluctuations, and in consequence, they have greater weight in the summation than would be the case if all the echoes were of equal power [29].

The behavior of the effective number of looks as a function of delay time, however, is dominated by the variation of the correlation function, as a comparison of Fig. 5 with Fig. 3 demonstrates. While the maximum effective number of looks occurs around zero delay time, it falls rapidly toward zero at around $60 \mathrm{~ns}$. The speckle suppression of the multilooking is poor at larger delay times. This is apparent in the noticeable increase in phase noise that is visible in the observations, as illustrated in Fig. 9, where the phase noise visibly increases at larger delay times.

There is a less agreement between prediction and observation at zero delay times, and at larger negative delays, although the causes of these are different. First, the theory in this section supposes that speckle noise is the only source of fluctuation. If there is a source of variation in the observations in addition to that due to speckle, the observed effective number of looks will be less than predicted. In particular, the echoes are very sensitive to the SWH in the vicinity of zero delay time. We formed the observed values of the effective number of looks by examining the along-track variances (the details are provided in Appendix B), and in consequence, the observations include whatever variations result from changes in the roughness along the track, whereas the theory has been evaluated at a fixed roughness. There is additional variability in the data that is not presented in the theory, and some differences between the two in the vicinity of zero delay are to be expected. 


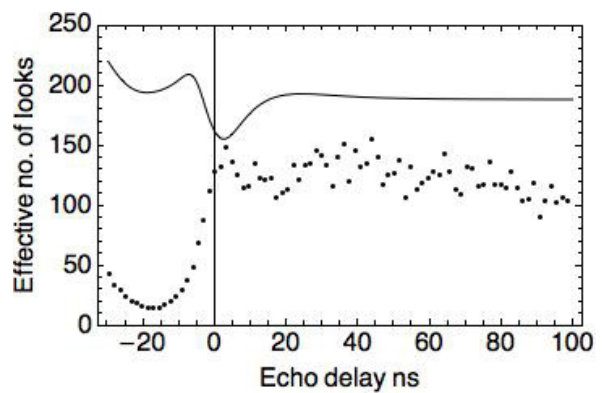

Fig. 6. Comparison of the calculated (solid line) and observed (dots) effective number of looks for SAR mode echoes at $20 \mathrm{~Hz}$. The calculated curve is for a wave height of $2 \mathrm{~m}$. The actual number of looks at $20 \mathrm{~Hz}$ is 240 .

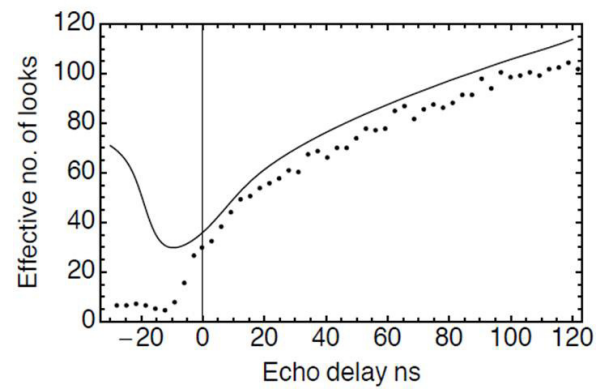

Fig. 7. Comparison of the calculated (solid line) and observed (dots) effective number of looks at $20 \mathrm{~Hz}$ of pulse-limited echoes observed in SAR mode. The calculated curve is for a wave height of $2 \mathrm{~m}$. The actual number of looks at $20 \mathrm{~Hz}$ is 256.

At larger negative delay times, the observations show greater variability than is predicted theoretically. We have not been able to identify precisely the source of this behavior, but for these values of delay time, the echo power is very small, and the estimate of the effective number of looks is the ratio of two very small numbers (the mean echo squared and the variance of the echo). Small errors in the theory arising from approximations used to obtain (4), for example, or small departures of the actual instrument from its theoretical description will result in significant differences in the comparison of theory with observations. However, because the echo powers are small, this behavior has little effect on the measurement precision of the parameters.

Fig. 6 shows the corresponding comparison for the SAR mode echo power, where the calculated values use (30). As argued in Section III-B, the echoes that contribute to the multilooked echo are independent; the difference between the actual and effective number of looks is, in theory, entirely the result of forming the looks from different statistical populations. Because all the looks are independent, there is a somewhat higher ratio of effective to actual looks, or around 180:240 in this case. In practice, the effective number of looks is somewhat smaller, around 140 looks, and as with the SARIN cross-products, the echoes in the vicinity of zero delay time have greater variability than the theory acknowledges.

Fig. 7 shows the comparison of the effective number of looks for the pulse-limited echoes, where the theoretical values are calculated from (34). In contrast to the SAR echoes, the pulse-limited echoes are drawn from the same statistical population, and the disparity between the actual and effective number of looks is entirely the result of correlations between the looks. Fig. 4 illustrates that at zero delay time, approximately only every fifth look can be considered independent for pulse-limited echoes at high wave-heights; this is apparent too from the ratio between the actual and effective number of looks illustrated in Fig. 7, in which the ratio is closer to $1: 6$. The effective number of looks improves at higher delay times, due to the greater spatial distances between the area illuminated at a given delay time.

Overall, however, the comparison between the actual and effective number of looks is reasonably close. Even in the case of the SAR mode echo powers, the ratio between the theoretical and measured number of looks is approximately 5:4. Thus, one may conclude that during its operation, the CryoSat-2 SIRAL radar is operating close to its theoretical limit in achieving speckle suppression.

\section{CONCLUSION}

In this paper, we have developed a model of the synthetic aperture, interferometric radar altimeter echo power and echo cross-product, and made the necessary simplifications, based on the smallness of the pitch and roll angles and the limited range of the satellite altitude to make the model applicable to large ocean data sets. We have shown that by simple modifications, the pulse-limited echo can be recovered as a simplification of the SAR mode echo power, demonstrating the equivalence of the SAR and pulse-limited cases. We determine the theoretical effective number of looks-a measure of the effectiveness of the multilooking in suppressing speckle fluctuations - in the SARIN and SAR modes, and have compared this with that provided by pulse-limited operation. We expect that the echo model will be of interest to the ocean operational altimetry community, especially during the transition from predominantly pulse-limited instruments to SAR instruments, when this model can be applied to both operating modes, especially the Sentinel-6 mission which is capable of forming SAR and pulse-limited echoes simultaneously via socalled "interleaved" operation [30], [41]-[43].

\section{APPENDIX A \\ CRYOSAT-2 DATA}

In this paper, two sets of acquisitions of CryoSat-2 data have been used. The results that depend on the measurements of the echo cross-product (Section III-E) were obtained from acquisitions of SIRAL SARIN mode data observed between July 27, 2010 and July 28, 2010 from the Pacific and Indian Oceans. The satellite tracks from which these are taken are a subset of those shown in [31, Fig. 2]. For this paper, the data acquisitions falling on orbit numbers $1595,1599,1601$, and 1607 were used. These data were processed using version Vk 1.0 of the L1b processor (used by ESA for the distribution of CryoSat data products from February 2012 to April, 2013), and the data we used were the SARIN mode L1b data product [39].

For the results that concern the SAR mode and pulse-limited echoes, the data we used were SIRAL SAR mode acquisitions 


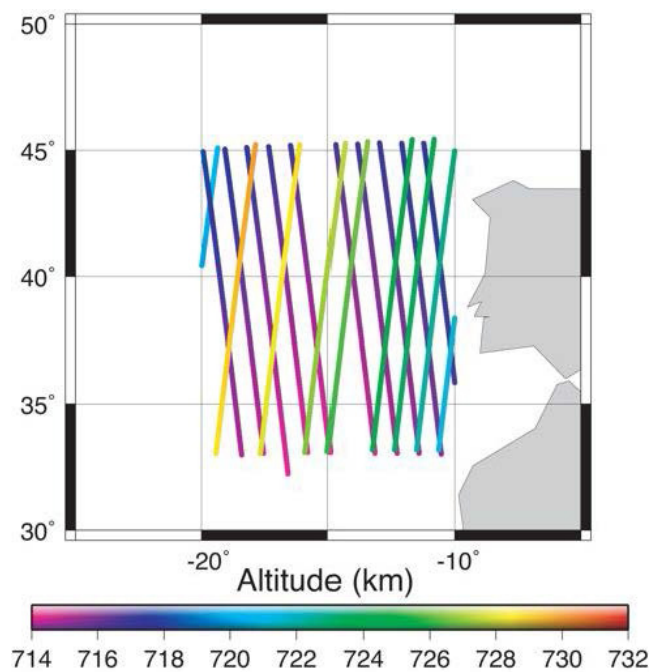

Fig. 8. Location and altitudes of the SAR mode acquisition used in this paper. The gray area on the right of the plot are the Iberian Peninsula and the northwest Africa.

observed from a region off the coast of Portugal in the North Atlantic between January 4, 2011 and January 29, 2011. This region was chosen by ESA mission management as providing for a wide range of ocean sea states while falling within the operational constraints of data storage and downlink capability. The location of the satellite tracks during these acquisitions is given in Fig. 8. These data were also processed using version $\mathrm{Vk} 1.0$ of the L1b processor, from which SAR mode L1b data were obtained [39].

In addition to the SAR L1b data, we also obtained from the same acquisitions of the set of so-called "CFBR" data. These data have the same format as the ESA full bit rate data [39] save that, in addition, they have had applied the full set of internal range, amplitude, phase, and phase difference corrections that are generated from the on-board internal calibration measurements. It is from the CFBR data that we obtained the "pulse-limited" data that is described in the main text.

In SAR mode, SIRAL uses a so-called "closed-burst" operation in which bursts of pulses are transmitted at intervals long enough that the echoes from earlier bursts can be received. A single burst consists of 64 phase coherent pulses with an individual PRF set at $18.182 \mathrm{kHz}$, which are received at the antenna at a burst-repetition frequency of $84.796 \mathrm{~Hz}$. (Each burst is of 3.52-ms duration, with an interval of $11.7 \mathrm{~ms}$ between the transmission of successive bursts.) It is these data, with internal calibrations applied and orbit and data information added, which form the CFBR data.

In the processing of SAR mode L1b data, the echoes received from each burst are processed by performing a "range fast Fourier transform (FFT)" (completing the pulse compression of the altimeter) and performing the so-called "azimuthal" FFT. The azimuthal FFT has the effect of focusing the echoes into 63 "beams" directed at consecutive strips on the surface. (While the FFT generates 64 "beams," one of these is at the Nyquist-Doppler frequency and cannot be unambiguously associated with either a forward- or backward-looking beam.) Echoes from successive bursts directed at the same strip on the surface are grouped, or "stacked," corrected for their slant range (sometimes termed "delay compensated"), incoherently detected, and then summed or "multilooked" to reduce the speckle noise. The number of nonzero beams or "number of looks" in each stack is approximately 240. (In detail, the number depends weakly on the altitude.) Each multilooked, "L1b" echo is present in the L1b data for each strip alongtrack. These are separated at approximately $300 \mathrm{~m}$ along the track, and at a satellite, ground velocity of $6700 \mathrm{~m} \cdot \mathrm{s}^{-1}$ (i.e., the speed with which the nadir point moves over earth's surface) occurs at a rate of approximately $21.4 \mathrm{~Hz}$. (A more detailed description of the processing applied to the data can be found in [23].) In SARIN mode, the burst rate is reduced by a factor of 4 compared with that of the SAR mode and there are 61 looks in the multilooked echo cross-product at $20 \mathrm{~Hz}$. The SARIN echo power, however, sums incoherently the power received on each antenna, and thus in this case, there are 122 looks.

If, however, the processing is interrupted following the range FFT, the burst consists of 64 echoes that have arisen from a conventional, pulse-limited geometry. These echoes can be incoherently detected, and summed, to provide a multilooked, pulse-limited echo at a rate of $85.5 \mathrm{~Hz}$. In our processing, we determine a "retracking" correction, using an "offset center of gravity" (OCOG) retracker [40], at an $85.5-\mathrm{Hz}$ rate. These corrections are smoothed with a Gaussian filter whose bandwidth is equivalent to $1 \mathrm{~Hz}$, and its outcome is used to align the $85.5-\mathrm{Hz}$ echoes, which are then further summed to generate multilooked, pulse-limited echoes at a rate of $21.4 \mathrm{~Hz}$. Each $21.4-\mathrm{Hz}$ echo is then the result of 256 looks. This data set then comprises the "pulselimited" echoes referred to in the main text. There is not a one-to-one correspondence between the "L1b" SAR mode echoes and the pulse-limited echoes, because the rate of the former is determined by the distance along-track that separates the strips, whereas the latter is determined by the burst timing. They are, however, surveying the same track over the ocean at the same time, and at a similar rate. For simplicity, we refer to both sets of echoes as occurring at $20 \mathrm{~Hz}$.

The theory of Section II assumes that either the pitch or the pitch and roll are small, and that the relative variations of the satellite altitude and velocity are small. Table I provides the range of these parameters for the two data acquisitions that we use. With regard to the pitch and roll angles reported in the L1b data products, these are known to be affected by static biases. We have applied a correction of $-0.1062^{\circ}$ to the roll angles [28] and of $-0.055^{\circ}$ to the pitch angles [31] to account for these biases.

\section{APPENDIX B \\ Model FitTing AND PostPRocessing OF CRYOSAT-2 DATA}

The calculation of the models of (21) and its SAR and pulse-limited equivalents were performed using the computational package Mathematica. The numerical integrations of (1), (10), and (13) were performed using the routine NIntegrate. Intermediate results [such as the functions $J$ 
TABLE I

Variations in Altitude, Altitude Rate, SATELlite SPEED, PITCH, AND ROLL

\begin{tabular}{|c|c|c|c|c|}
\hline Altitude & $\begin{array}{c}\text { Altitude } \\
\text { rate } \\
\left(\mathrm{m} \mathrm{s}^{-1}\right)\end{array}$ & $\begin{array}{c}\text { Satellite } \\
\text { speed } \\
\left(\mathrm{km} \mathrm{s}^{-1}\right)\end{array}$ & Pitch & Roll \\
$(\mathrm{km})$ & degrees $)$ & (degrees) \\
\hline \multicolumn{6}{|c|}{ SAR Mode Acquisitions } \\
\hline $714-$ & $-7.50-$ & $7.52-$ & $-0.7--$ & $-0.15-$ \\
730 & 17.0 & 7.54 & 0.02 & 0.01 \\
\hline \multicolumn{5}{|c|}{ SARIN Mode Acquisitions } \\
\hline $721-$ & $-20.2-$ & $7.51-$ & $-0.02-$ & $-0.09-$ \\
735 & 21.2 & 7.53 & 0.05 & 0.32 \\
\hline
\end{tabular}

TABLE II

Values of the System Parameters Used in the Model of the Echo Cross-Product ANd Echo Power

\begin{tabular}{|c|c|c|}
\hline Parameter & Symbol & Value \\
\hline Speed of light & $c$ & $299,792,458 \mathrm{~m} \mathrm{~s}^{-1}$ \\
\hline Satellite altitude & $h_{0}$ & $720 \mathrm{~km}$ \\
\hline Altitude variation & $\delta$ & Variable $^{(1)}$ \\
\hline Antennas power gain & $G_{0}$ & $42 \mathrm{~dB}$ \\
\hline Earth radius & $R$ & $6380 \mathrm{~km}$ \\
\hline Geometric factor & $\eta$ & 1.113 \\
\hline Synthetic beam gain & $D_{0}$ & 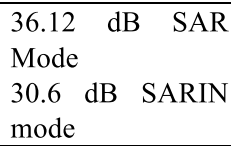 \\
\hline Carrier wavelength & $\lambda$ & $0.022 \mathrm{~m}$ \\
\hline Carrier wavenumber & $k_{0}$ & $285.5 \mathrm{~m}^{-1}$ \\
\hline Interferometer baseline & $B$ & $1.1676 \mathrm{~m}$ \\
\hline $\begin{array}{l}\text { Along-track antenna } \\
\text { parameter }\end{array}$ & $\gamma_{1}$ & 0.0116 radians \\
\hline $\begin{array}{l}\text { Across-track antenna } \\
\text { parameter }\end{array}$ & $\gamma_{2}$ & 0.0129 radians \\
\hline Pitch & $\mu$ & Variable $^{(1)}$ \\
\hline Roll & $\chi$ & Variable $^{(1)}$ \\
\hline Satellite speed & $v_{s}$ & $7.53 \mathrm{~km} \mathrm{~s}^{-1}$ \\
\hline $\begin{array}{l}\text { Number of samples in } \\
\text { synthetic aperture }\end{array}$ & $N_{a}$ & 64 \\
\hline Burst repetition interval & $\Delta_{b}$ & $\begin{array}{lll}11.7 & \mathrm{~ms} & \mathrm{SAR} \\
\text { mode } & & \\
46.7 \mathrm{~ms} & \text { SARIN } \\
\text { mode } & & \end{array}$ \\
\hline Pulse repetition interval & $\Delta_{t}$ & $55 \mu \mathrm{s}$ \\
\hline Number of looks at $20 \mathrm{~Hz}$ & $\begin{array}{c}N_{S} \\
N_{S I} \\
N_{P}\end{array}$ & $\begin{array}{l}240 \text { SAR mode } \\
61 \text { SARIN mode } \\
256 \text { pulse-limited }\end{array}$ \\
\hline
\end{tabular}

in (13)] and the final numerical functions [the functions $G$ of (21)] were formed by applying the routine interpolation to the tabular outcome of the previous numerical integration. We established by trial and error that the sampling of the tabular outcome of each integration step was sufficient that the outcome of the succeeding integration differed less than $0.25 \%$ when the sampling was further densified. In general, we found that 0.1-ns sampling was sufficient.

The functions $\Psi_{r}\left(\tau-\tau_{0}\right)$ and $p_{r}\left(\tau-\tau_{0}\right)$ were fit to the echo cross-product and echo powers, respectively. The free parameters in the fit were the set $\left\{\tau_{0}, \sigma_{s}, \sigma^{0}, \theta\right\}$ in the case of the echo cross-product, and $\left\{\tau_{0}, \sigma_{s}, \sigma^{0}\right\}$ in the case of the SAR and pulse-limited powers. The values of other parameters that were used in the models are given in Table II. To fit the echoes, we first nondimensionalized the echoes using the echo duration and echo amplitude as scale parameters in order that the nondimensionalized echoes were $O(1)$ in range and amplitude. The nondimensionalized parameters were chosen so as to minimize the weighted least-squares difference between the model and the echo, using as weights the reciprocal of the variances whose determination is described below. In the case of the powers, we used the routine NonLinearModelFit to perform the fit (using the Levenberg Marquardt algorithm); in the case of the cross-product, we formed the square of the amplitude of the (complex) difference between the crossproduct and the model and used the routine FindMinimum to determine the parameters. (We did this because the Mathematica routine NonLinearModelFit does not accommodate complex functions. We established by using this method to fit an example of a (real) power echo that the two routines returned the same answer for the fit parameters.) Fig. 9 shows an example of a fit to a $20-\mathrm{Hz}$ echo cross-product, presented in an amplitude and phase representation.

The variances that we used to weight the fit are those of (26). These variances are those of the crossproduct or power at fixed values of the echo delay $\tau$. To estimate them from the measurement echoes, the echoes need to be aligned, so that averages are taken across constant values of the echo delay. The shift in delay of the observed echoes needed to achieve this is the value of that arises from the fit, but in order to obtain this accurately, the weights, and thus variances, must first be determined. Here again, we use an iterative approach, in which we align the echoes using an OCOG retracker [40] to obtain a retracking correction at $20 \mathrm{~Hz}$. The sequence of retracking corrections is then smoothed with a Gaussian filter with a half width equal to $1 \mathrm{~s}$ to generate a set of smoothed retracking corrections at $20 \mathrm{~Hz}$. These smoothed corrections are then applied to the $20-\mathrm{Hz}$ data to align them to constant delay time reference. (This smoothing is performed to reduce the noise on the correction resulting from speckle.) Having done that, we then formed estimates of the mean echo, and the variance about the mean, as a function of delay time $\tau$. The variances obtained this way were used to provide weights for the fitting routines, which generated a further sequence of precise retracking corrections at $20 \mathrm{~Hz}$ from the values of $\tilde{\tau}_{0}$ obtained from the fit.

This sequence was again smoothed over $1 \mathrm{~Hz}$, and the result reapplied to determine the final sequence of echoes from which the effective number of looks plotted in Section III-E was obtained. (In practice, we did not find that the additional accuracy provided by using the sequence of values of $\tilde{\tau}_{0}$ made a significant difference to the effective number of looks.)

We applied postprocessing editing on the along-track results through " $3-\sigma$ " editing of the results by passing them through a 10-s smoothing filter and removing outliers whose residual from the smoothed results exceeded three standard deviations of the along-track residual distribution. We also removed values for which the least-squares fitting failed to converge. The success with which convergence was achieved was sensitive to the initial values provided to the fit routine as a 

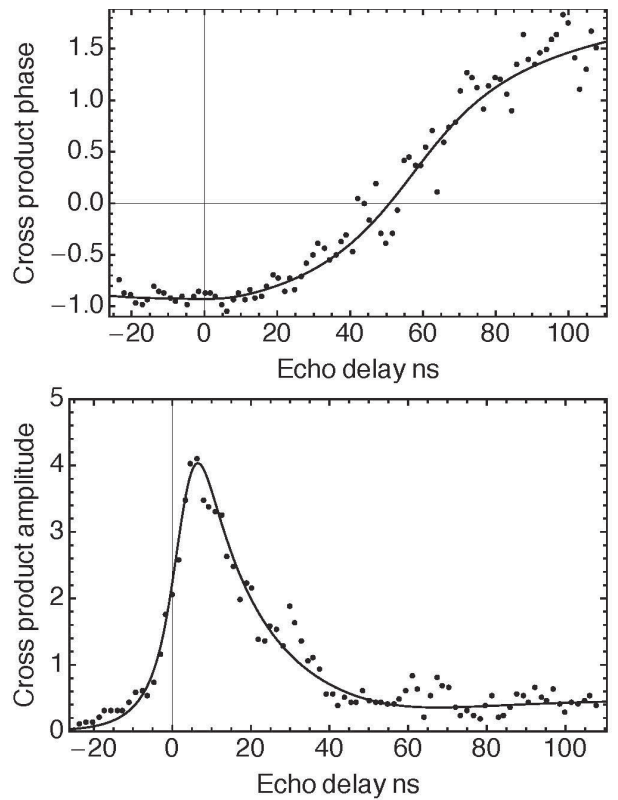

Fig. 9. Example of a fit to a $20 \mathrm{~Hz}$, SARIN mode, echo cross-product. The fit parameters are $\tilde{\tau}_{0}=1.355 \mathrm{~ns}, \tilde{\sigma}_{s}=0.456 \mathrm{~m}$, and $\tilde{\theta}=0.1638^{\circ}$. Note that the cross-product phase at zero delay is approximately equal to $k_{0} B \theta$ or $0.966 \mathrm{rad}$ $\left(55.35^{\circ}\right)$ in this case. The increase in phase noise at larger delay times-a consequence of the behavior of the cross-product coherence-is visible in the figure.

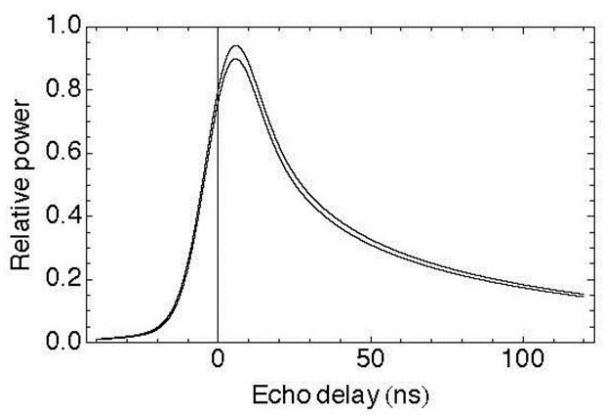

Fig. 10. Comparison of the SAR mode or SARIN mode mean echo power that occurs with an altitude rate of zero and that with an altitude rate of $40 \mathrm{~m} \cdot \mathrm{s}^{-1}$. When the altitude rate is nonzero, the outer beams are lost from the summation in the multilooking, resulting in a lower power, and some alteration of the echo shape. The maximum difference is some $4 \%$. The calculation is done for a wave height of $2 \mathrm{~m}$.

starting point for its iteration, particularly those of $\tilde{\tau}_{0}$ and $\tilde{\sigma}_{s}$. We improved on the success rate by repeating the fits using the smoothed values as a second set of initial values. Following this pass, some $6 \%$ of the values we obtained were removed by editing.

To obtain the $1-\mathrm{Hz}$ values of precision described in the text, we separated the sequence of fit values into 1-s blocks. We fit a straight line through each $1-\mathrm{s}$ set of $20-\mathrm{Hz}$ values, and took the standard deviation of the residuals as the measurement precision. While there are alternatives to this piecewise approach, this method is conventional (R. Scharroo, personal communication) in reporting altimeter precisions.

Details of the method and external data sets used to perform the calibration of the interferometer whose result is shown in Fig. 9 are given in [28].

\section{APPENDIX C \\ EFFECT OF ALTITUDE RATE ON SARIN AND SAR MODE ECHO SHAPE}

As noted in the main text, when the satellite velocity vector is not tangential to the surface at the nadir point, the distribution of beam look angles is not symmetric relative to the nadir direction, but around the normal to the velocity vector. Because the synthetic beam gain is modulated by the antenna gain, beams that are formed at angles larger than would otherwise be the case are strongly attenuated by the antenna gain, and accounting for them by extending the summation to include them has little effect on the summation of (3). However, there is a corresponding loss of beams at angles smaller than those included in the summation of (3), and if the altitude rate of the satellite is sufficiently high, the loss of power in the multilooked echo is sufficient to alter the echo shape in a detectable way. Fig. 10 shows the effect on the SAR mode echo shape of an altitude rate of $40 \mathrm{~m} \cdot \mathrm{s}^{-1}$. With this altitude rate, the normal to the satellite velocity makes an angle of $0.3^{\circ}$ with the nadir direction, and some ten beams are lost from the summation of (3). The effect increases exponentially with altitude rate, because of the modulation of the antenna pattern. For altitude rates smaller than $20 \mathrm{~m} \cdot \mathrm{s}^{-1}$, the effect is negligible. In this paper, the maximum altitude rate that occurred during the acquisitions was $21.2 \mathrm{~m} \cdot \mathrm{s}^{-1}$ (Table I) and this rate was rarely encountered, and we have not accounted for this effect in the models used to fit the data described in this paper. The expected effect on estimated backscatter is small, as the backscatter estimate is dominated by the AGC setting and not the fit waveform amplitude.

\section{ACKNOWLEDGMENT}

Many of the results in this paper were presented at the 2011 OSTST Meeting (October 19-21, 2011, San Diego, CA, USA). The authors would like to thank L. Phalippou and C. Gommenginger for their helpful criticism and advice. The participation of W. H. F. Smith does not constitute a statement of policy, decision, or position on behalf of the National Oceanic and Atmospheric Administration or the U.S. Government.

\section{REFERENCES}

[1] J. G. Marsh and T. V. Martin, "The SEASAT altimeter mean sea surface model," J. Geophys. Res., vol. 87, no. C5, pp. 3269-3280, 1982.

[2] D. T. Sandwell and W. H. F. Smith, "Marine gravity anomaly from Geosat and ERS 1 satellite altimetry," J. Geophys. Res., vol. 102, no. B5, pp. 10039-10054, 1997.

[3] L. Miller and R. Cheney, "Large-scale meridional transport in the tropical Pacific Ocean during the 1986-1987 El Niño from Geosat," J. Geophys. Res., vol. 95, no. C10, pp. 17905-17919, 1990.

[4] R. E. Cheney and J. G. Marsh, "Seasat altimeter observations of dynamic topography in the Gulf Stream region," J. Geophys. Res., vol. 86, no. C1, pp. 473-483, Jan. 1981.

[5] C. Wunsch and D. Stammer, "The global frequency-wavenumber spectrum of oceanic variability estimated from TOPEX/POSEIDON altimetric measurements," J. Geophys. Res., vol. 100, no. C12, pp. 24895-24910, 1995.

[6] D. E. Cartwright and R. D. Ray, "Oceanic tides from Geosat altimetry," J. Geophys. Res., vol. 95, no. C3, pp. 3069-3090, 1990.

[7] C. L. Rufenach and W. R. Alpers, "Measurement of ocean wave heights using the Geos 3 altimeter," J. Geophys. Res., vol. 83, no. 10, pp. 5011-5018, 1978. 
[8] N. M. Mognard and B. Lago, "The computation of wind speed and wave heights from Geos 3 data," J. Geophys. Res., vol. 84, no. 8, pp. 3979-3986, 1979.

[9] L.-L. Fu and A. Cazenave, Satellite Altimetry and Earth Sciences, a Handbook of Techniques and Applications (International Geophysics Series), vol. 69, R. Dmowska, J. R. Holton, and H. T. Rossby, Eds. San Diego, CA, USA: Academic, 2001.

[10] D. B. Chelton, J. C. Ries, B. J. Haines, L.-L. Fu, and P. S. Callahan, "Satellite altimetry," in Satellite Altimetry and Earth Sciences, a Handbook of Techniques and Applications (International Geophysics Series), vol. 69, L.-L. Fu and A. Cazenave, Eds. San Diego, CA, USA: Academic, 2001

[11] J. Lambin et al., "The OSTM/jason-2 mission," Marine Geodesy, vol. 33, pp. 4-25, Aug. 2010.

[12] K.-R. Choi, J. C. Ries, and B. D. Tapley, "Jason-1 precision orbit determination by combining SLR and DORIS with GPS tracking data," Marine Geodesy, vol. 27, nos. 1-2, pp. 319-331, 2004.

[13] B. Haines, Y. Bar-Sever, W. Bertiger, S. Desai, and P. Willis, "Onecentimeter orbit determination for Jason-1: New GPS-based strategies," Marine Geodesy, vol. 27, nos. 1-2, pp. 299-318, 2004.

[14] P. C. Marth et al., "Prelaunch performance of the NASA altimeter for the TOPEX/Poseidon project," IEEE Trans. Geosci. Remote Sens., vol. 31 , no. 2, pp. 315-332, Mar. 1993.

[15] M. Roca, S. Laxon, and C. Zelli, "The EnviSat RA-2 instrument design and tracking performance," IEEE Trans. Geosci. Remote Sens., vol. 47, no. 10 , pp. 3489-3506, Oct. 2009.

[16] P. Raizonville, N. Lannelongue, J. C. Anne, and P. de Chateau Thierry, "POSEIDON solid state altimeter," Acta Astronau., vol. 17, no. 1, pp. 23-30, 1988.

[17] J. T. McGoogan, "Satellite altimetry applications," IEEE Trans. Microw. Theory Tech., vol. MTT-23, no. 12, pp. 970-978, Dec. 1975.

[18] J. MacArthur, "Design of the SEASAT-A radar altimeter," in Proc. Oceans Conf., Washington, DC, USA, Sep. 1976, pp. 222-229.

[19] G. S. Brown, "The average impulse response of a rough surface and its applications," IEEE Trans. Antennas Propag., vol. AP-25, no. 1, pp. 67-74, Jan. 1977.

[20] D. J. Wingham, A. J. Ridout, R. Scharroo, R. J. Arthern, and C. K. Shum, "Antarctic elevation change from 1992 to 1996," Science, vol. 282, no. 5388, pp. 456-458, 1998.

[21] H. J. Zwally, R. A. Bindschadler, A. C. Brenner, J. A. Major, and J. G. Marsh, "Growth of Greenland ice sheet: Measurement," Science. vol. 246, no. 4937, pp. 1587-1589, 1989.

[22] S. W. Laxon, N. Peacock, and D. Smith, "High interannual variability of sea ice thickness in the Arctic region," Nature, vol. 425, pp. 947-950, Oct. 2003

[23] D. J. Wingham et al., "CryoSat: A mission to determine the fluctuations in Earth's land and marine ice fields," Adv. Space Res., vol. 37, no. 4, pp. 841-871, 2006.

[24] W. T. K. Johnson, "Magellan imaging radar mission to Venus," Proc IEEE, vol. 79, no. 6, pp. 777-790, Jun. 1991.

[25] J. R. Jensen, "Angle measurement with a phase monopulse radar altimeter," IEEE Trans. Antennas Propag., vol. 47, no. 4, pp. 715-724, Apr. 1999.

[26] R. K. Raney, "The delay/Doppler radar altimeter," IEEE Trans. Geosci. Remote Sens., vol. 36, no. 5, pp. 1578-1588, Sep. 1998.

[27] J. R. Jensen and R. K. Raney, "Delay/Doppler radar altimeter: Better measurement precision," in Proc. IEEE Geosci. Remote Sens. Symp. (IGARSS), Seattle, WA, USA, Jul. 1998, pp. 2011-2013.

[28] N. Galin, D. J. Wingham, R. Cullen, M. Fornari, W. H. F. Smith, and S. Abdalla, "Calibration of the CryoSat-2 interferometer and measurement of across-track ocean slope," IEEE Trans. Geosci. Remote Sens., vol. 51, no. 1, pp. 57-72, Jan. 2013.

[29] D. J. Wingham, L. Phalippou, C. Mavrocordatos, and D. Wallis, "The mean echo and echo cross product from a beamforming interferometric altimeter and their application to elevation measurement," IEEE Trans. Geosci. Remote Sens., vol. 42, no. 10, pp. 2305-2323, Oct. 2004

[30] R. K. Raney, "CryoSat SAR-mode looks revisited," IEEE Trans. Geosci. Remote Sens Lett., vol. 9, no. 3, pp. 393-397, May 2012.

[31] N. Galin, D. J. Wingham, R. Cullen, R. Francis, and I. Lawrence, "Measuring the pitch of CryoSat-2 using the SAR mode of the SIRAL altimeter," IEEE Geosci. Remote Sens. Lett., vol. 11, no. 8, pp. 1399-1403, Aug. 2014.

[32] D. J. Wingham and D. W. Wallis, "The rough surface impulse response of a pulse-limited altimeter with an elliptical antenna pattern," IEEE Antennas Propag. Lett., vol. 9, pp. 232-235, 2010.
[33] G. S. Hayne, "Radar altimeter mean return waveforms from near-normalincidence ocean surface scattering," IEEE Trans. Antennas Propag., vol. AP-28, no. 5, pp. 687-692, Sep. 1980.

[34] P. Beckmann and A. Spizzichino, The Scattering of Electromagnetic Waves From Rough Surfaces, vol. 4. Oxford, U.K.: Pergammon Press, 1963, p. 503.

[35] M. V. Berry, "The statistical properties of echoes diffracted from rough surfaces," Philos. Trans. Roy. Soc. London A, Math. Phys. Sci., vol. 273 , no. 1237 , pp. 611-654, 1973.

[36] E. Rodriguez and J. M. Martin, "Correlation properties of ocean altimeter returns," IEEE Trans. Geosci. Remote Sens., vol. 32, no. 3, pp. 553-561, May 1994.

[37] E. J. Walsh, "Analysis of experimental NRL radar altimeter data," Radio Sci., vol. 9, nos. 8-9, pp. 711-722, Aug./Sep. 1974.

[38] E. J. Walsh, "Pulse-to-pulse correlation in satellite radar altimeters," Radio Sci., vol. 17, no. 4, pp. 786-800, Jul./Aug. 1982.

[39] CryoSat Product Handbook. [Online]. Available: http://emits.esa.int/ emits-doc/ESRIN/7158/CryoSat-PHB-17apr2012.pdf

[40] D. J. Wingham, C. G. Rapley, and H. Griffiths, "New techniques in satellite altimeter retracking systems," in Proc. IGARSS Symp., Zürich, Switzerland, 1986, pp. 1339-1344

[41] R. K. Raney, "Maximizing the intrinsic precision of radar altimetric measurements," IEEE Geosci. Remote Sens. Lett., vol. 10, no. 5, pp. 1171-1174, Sep. 2013

[42] C. Gommenginger, C. Martin-Puig, L. Amarouche, and R. K. Raney, "Review of state of knowledge for SAR altimetry over ocean," Nat. Oceanogr. Centre, Southampton, U.K. Tech. Rep. EUM/RSP/REP/14/749304, Version 2.2, 2013, p. 57.

[43] F. Boy, J.-D. Desjonquéres, N. Picot, T. Moreau, and M. Raynal, "CryoSat-2 SAR-mode over oceans: Processing methods, global assessment, and benefits," IEEE Trans. Geosci. Remote Sens., vol. 55, no. 1, pp. 148-158, Jan. 2016, doi: 10.1109/TGRS.2016.2601958.

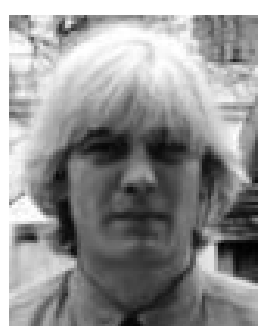

Duncan J. Wingham received the B.Sc. degree in physics from the University of Leeds, Leeds, U.K., in 1979, and the Ph.D. degree in physics from the University of Bath, Bath, U.K., in 1984.

In 1986, he joined the University College London (UCL), London, U.K., where he held lecturing posts at the Mullard Space Science Laboratory and at the Department of Electronic and Electrical Engineering. In 1996, he was appointed to a Chair in the Department of Space and Climate Physics, and from 2005 to 2010, he was the Head of the Department of Earth Sciences, UCL. From 2000 to 2012, he was a Lead Investigator of the ESA CryoSat and CryoSat-2 satellite missions. Since 2012, he has been a Chief Executive of the United Kingdom Natural Environment Research Council.

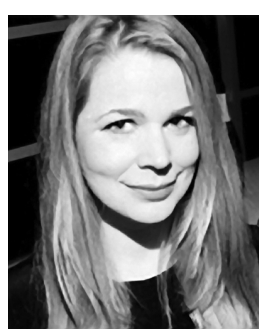

Katharine A. Giles received the M.Sc. degree in earth and space science and the Ph.D. degree in space and climate physics from University College London (UCL), London, U.K., in 2000 and 2005, respectively.

She earned a U.K. Natural Environment Research Council Independent Research Fellowship, working with the Centre for Polar Observation and Modelling, Department of Earth Sciences, UCL, on problems of measuring sea-surface height and sea-ice thickness in the polar oceans. In 2013, she was awarded a lectureship by the Department of Earth Sciences, UCL. She was tragically killed in a road traffic accident in London on April 8, 2013.

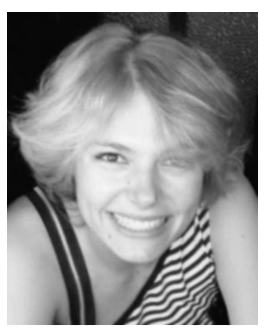

Natalia Galin received the B.Eng. degree in electrical engineering from the University of New South Wales, Sydney, NSW, Australia, in 2006. She submitted her Ph.D. dissertation "Snow Thickness Estimation Over Antarctic Sea Ice Using Helicopter Borne 2-8 GHZ FMCW Radar" to the University of Tasmania, Hobart, TAS, Australia.

In 2009, she joined the NASA/Goddard Space Flight Center, Greenbelt, MD, USA, as a Visiting Postgraduate Fulbright Scholar. She held research positions at the Department of Earth Sciences, University College London, London, U.K., and at the NOAA Laboratory for Satellite Altimetry, Greenbelt, MD, USA, where she focused on CryoSat-2. 


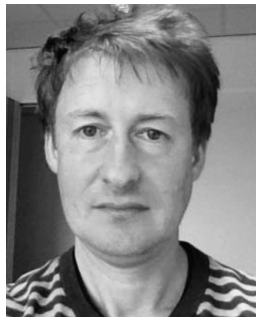

Robert Cullen received the B.Sc. degree in electrical engineering from the University of Hull, Hull, U.K., the M.Sc. degree in satellite communications and spacecraft technology from the University College London (UCL), London, U.K., and the Ph.D. degree in geophysics from the University of Aston, Birmingham, U.K.

In 1998, he joined the Department of Space and Climate Physics, UCL, where he developed ESA ENVISAT and CryoSat mission simulation and processing algorithm capability. In 2003, he joined Vega Space Ltd., Luton, U.K., to support CryoSat commissioning and validation preparation activities. He became the CryoSat-2/SIRAL-2 Instrument Manager and went on to perform key roles in the mission preparation and execution. Since 2010, he has been the System and Payload Manager for the Jason-CS/Sentinel-6 ocean surface topography mission on behalf of a consortium including NASA, ESA, CNES, Eumetsat, and NOAA. Since 2014, he has also been supporting the development of future polar altimeter capability.

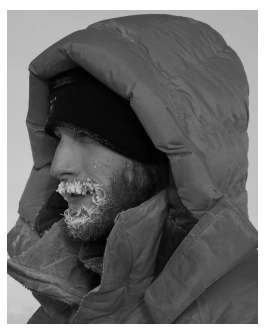

Thomas W. K. Armitage received the M.Sc. degree in physics and the Ph.D. degree in polar remote sensing from University College London (UCL), London, U.K., in 2011 and 2016, respectively.

From 2011 to 2012, he was a Young Graduate Trainee with the European Space Agency, Noordwijk, The Netherlands, focusing on novel interferometric CryoSat-2 retrievals over sea ice. He was a Research Associate with the Centre for Polar Observation and Modelling, Department of Earth Sciences, UCL. In 2017, he joined the Jet Propulsion Laboratory, California Institute of Technology, Pasadena, CA, USA.

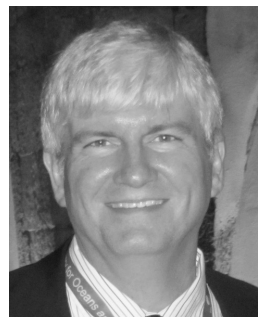

Walter H. F. Smith received the B.Sc. degree in geological science from the University of Southern California, Los Angeles, CA, USA, in 1984, and the M.A. and Ph.D. degrees in geological science from Columbia University, New York, NY, USA, in 1986 and 1990, respectively.

From 1984 to 1990, he was a Graduate Research Assistant with Columbia University. From 1990 to 1992, he was a Cecil and Ida Green Foundation Scholar with the Institute of Geophysics and Planetary Physics, Scripps Institution of Oceanography, La Jolla, CA, USA. Since 1992, he has been a Geophysicist with the National Oceanic and Atmospheric Administration (NOAA), College Park, MD, USA He served on the United Nations Committee for the General Bathymetric Chart of the Oceans for more than 20 years. He was a Principal Investigator on the Altimetric Bathymetry from Surface Slopes mission proposal for a delay/Doppler radar altimeter mission to map earth's oceans.

Dr. Smith is a fellow of the American Geophysical Union. He was a recipient of the U.S. Department of Commerce Gold Medal for scientific breakthroughs in the application of altimetry to bathymetric estimation, the NOAA Administrator's Award for rescuing and reprocessing data from the U.S. Navy Geosat mission, the NOAA Administrator's Award for scientific excellence and leadership in developing near-real-time marine and hurricane forecast system data from the European Space Agency CryoSat-2 mission, and the NESDIS STAR Technology Innovation Award for developing fully focused synthetic aperture radar altimetry. 